kristallinen Material als Gruppe von 7 Linien erscheinenden Spektrums der ungepaarten Elektronen zwischen den $\mathrm{Ga}$-Atompaaren möglich sein, welches entsprechend den beiden vorkommenden Ga-Isotopen aus 29 Einzellinien bestehen müßte.

Zur Beurteilung der Frage, durch welche Verunreinigungen die Bildung stabiler Defekte begünstigt wird, müßte wesentlich reineres Ausgangsmaterial zur Verfügung stehen. Auf Grund der bisherigen
Untersuchungen kann nur gesagt werden, daß auch bei $\mathrm{GaP}$ - ebenso wie bei $\mathrm{Si}$ - den Verunreinigungen eine entscheidende Bedeutung bei der Bildung von Strahlungsdefekten zukommt.

Für die Überlassung der bestrahlten Proben bin ich den Herren Bäuerlein und Dr. Wohlleben zu Dank verpflichtet. Herrn Schnitzke danke ich für die sorgfältige Durchführung der Messungen.

\title{
Magnetische, elektrische und thermische Eigenschaften von $\mathrm{FeGa}_{1,3}$
}

\author{
H. WAGINI \\ Forschungslaboratorium der Siemens-Schuckertwerke AG, Erlangen \\ (Z. Naturforschg. 21 a, 528-530 [1966] ; eingegangen am 2. Februar 1966) \\ Herrn Prof. Dr. Trendelenburg zum 70. Geburtstag gewidmet
}

\begin{abstract}
An der ferromagnetischen Verbindung $\mathrm{FeGa}_{1,3}$ wurde die Sättigungsmagnetisierung, der elektrische Widerstand, die absolute differentielle Thermospannung und die Wärmeleitfähigkeit oberhalb Zimmertemperatur gemessen. Es ergaben sich zwei Curie-Punkte bei $483^{\circ} \mathrm{K}$ und $697{ }^{\circ} \mathrm{K}$, die verschiedenen, gleichzeitig vorhandenen ferromagnetischen Strukturen zuzuordnen sind.
\end{abstract}

Unter den Schwermetallphasen, die mit III - VHalbleitern Eutektika bilden, findet man nach Untersuchungen von MüLler und WILHELM ${ }^{1}$ als neue ferromagnetische Verbindung $\mathrm{FeGa}_{1,3}$. Das $\mathrm{Zu}$ standsdiagramm des Systems $\mathrm{Fe}-\mathrm{Ga}$ wurde erst kürzlich von Dasarathy u. a. ${ }^{2}$ sowie Meissner u. a. ${ }^{3}$

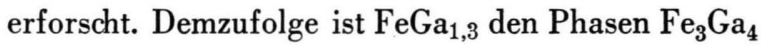
bzw. $\mathrm{Fe}_{8} \mathrm{Ga}_{11}$ gleichzusetzen. Die unterschiedlichen Stöchiometrien sind durch die Phasenbreite (ca. 56,6 bis 59,3 Atom-Proz. $\mathrm{Ga}^{2}$ ) bedingt. Die deutschen Autoren ${ }^{3}$ berichteten von einer monoklinen, C-flächenzentrierten Zelle, die Engländer ${ }^{2}$ konnten die Pulveraufnahmen tetragonal indizieren. Der Schmelzpunkt wurde mit ca. $906{ }^{\circ} \mathrm{C}$ und die Dichte mit $7,6 \mathrm{~g} / \mathrm{cm}^{3}$ angegeben. Weitere Daten sind bisher über $\mathrm{FeGa}_{1,3}$ nicht bekannt. Die vorliegende Arbeit berichtet über magnetische, elektrische und thermische Untersuchungen.

\section{Experimentelle Anordnung, Ergebnisse und Diskussion}

Obwohl die einkristallinen $\mathrm{FeGa}_{1,3}$-Einschlüsse aus dem eutektischen Gefüge isoliert werden kön-

1 A. Müller u. M. Wilhelm, J. Phys. Chem. Solids 26, 2029 [1965].

2 C. Dasarathy u. W. Hume-Rothery, Proc. Roy. Soc. London A 286, 141 [1965]. nen ${ }^{1}$, sind sie infolge ihrer geringen Abmessungen (maximale Länge $500 \mu$ ) den meisten physikalischen Messungen nicht zugänglich. Es wurde daher ein größeres Stück einer $\mathrm{Fe}-\mathrm{Ga}$-Legierung der Bruttozusammensetzung $\mathrm{FeGa}_{1,3}$ hergestellt. $\mathrm{Ga}(99,99 \%)$ und im $\mathrm{H}_{2}$-Strom geglühtes Carbonyleisen wurden im Atomverhältnis 1,3:1 zusammengeschmolzen und anschließend zum schnellen, ungerichteten Erstarren gebracht. Die metallographische Untersuchung des Schliffbildes zeigte mehrere Phasen. Nach mehrtägigem Tempern im Vakuum bei $800^{\circ}$ war das Material einphasig. Auch röntgenographisch waren die Proben den eutektischen Nadeleinschlüssen gleichwertig. Leider wies die Legierung kleine Poren auf, so daß die Meßergebnisse nur grobe Absolutwerte lieferten. Lediglich die geometrieunabhängige Thermospannung ist von diesen Fehlern nicht betroffen.

Für die magnetischen Untersuchungen wurde eine Probe $1,7 \mathrm{~mm} \times 1,7 \mathrm{~mm} \times 90 \mathrm{~mm}$ verwendet. Die Hysteresekurven wurden bei verschiedenen Temperaturen und $50 \mathrm{~Hz}$ mit der früher ${ }^{4}$ beschriebenen Apparatur oszillographisch aufgenommen. Auf diese Weise konnte nur die Anfangssuszeptibilität ermittelt

3 H. G. Meissner u. K. Schubert, Z. Metallk. 56, 523 [1965].

${ }^{4}$ H. W AGINI u. M. Wilhelm, Z. Naturforschg. 21 a, 329 [1966]. 
werden, da die erreichbare Luftinduktion von $2 \mathrm{kG}$ noch nicht zur Sättigung führte. Die Anfangssuszeptibilität zeigte als Funktion der Temperatur deutlich zwei Stufen: Der erste Abfall der Anfangssuszeptibilität lag oberhalb $400{ }^{\circ} \mathrm{K}$. Bei $697^{\circ} \mathrm{K}$ verschwand der Ferromagnetismus fast vollständig. Darüber war noch ein sehr schwacher Ferromagnetismus festzustellen.

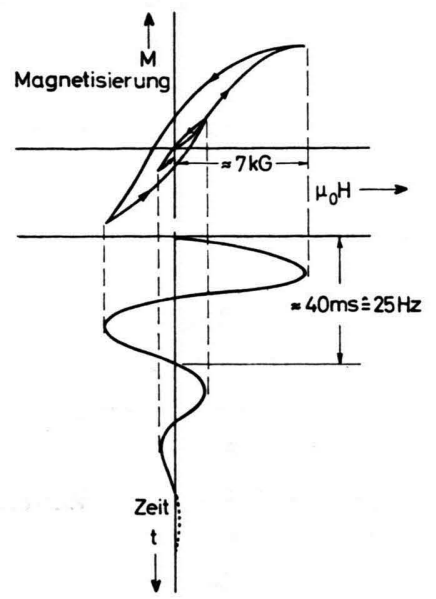

Abb. 1. Schematische Magnetisierungskurve von $\mathrm{FeGa}_{1,3}$ im gepulsten Magnetfeld.

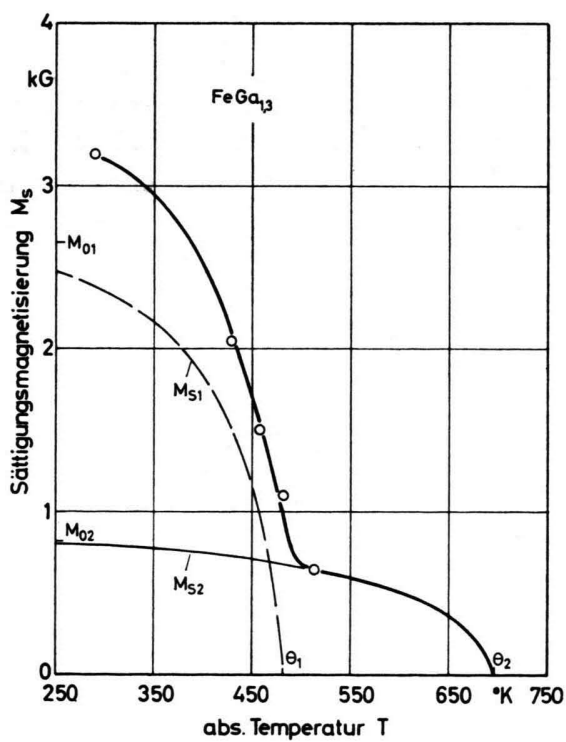

Abb. 2. Sättigungsmagnetisierung $\left(M_{\mathrm{s}}=B_{\mathrm{s}}-\mu_{0} H\right)$ von FeGa 1,3 als Funktion der absoluten Temperatur $T$. ○ Meßpunkte, - Gesamtmagnetisierung, $--M_{\mathrm{S} 1}$ : Sättigungsmagnetisierung der ferromagnetischen Phase 1 , $M_{\mathrm{s} 2}$ : Sättigungsmagnetisierung der ferromagnetischen Phase 2, $M_{01}, M_{02}$ extrapolierte Sättigungsmagnetisierungen für $T=0$.
Um eine von Verspannungen unabhängig magnetische Materialeigenschaft zu erhalten, mußte die Sättigung erreicht werden. Dies war ohne die Spule zu überlasten nur im Impulsbetrieb möglich. Eine auf ca. 2,5 kV aufgeladene Kondensatorbatterie von $400 \mu \mathrm{F}$ wurde über die Feldspule entladen. Im Maximum der gedämpften Schwingung von $25 \mathrm{~Hz}$ flossen ca. 130 A entsprechend einer Luftinduktion von $7 \mathrm{kG}$. Die Hysteresekurve, wie sie in Abb. 1 schematisch dargestellt ist, wurde in der üblichen Weise ${ }^{4}$ aufgezeichnet. Die gemessene Sättigungsmagnetisierung $\left(M_{\mathrm{s}}=B_{\mathrm{s}}-\mu_{0} H\right)$ ist in Abb. 2 als Funktion der absoluten Temperatur eingetragen (Meßgenauigkeit $\pm 200 \mathrm{G})$. Man erkennt wieder zwei Stufen.

Zur Interpretation dieses Sachverhaltes betrachten wir Abb. 3 oben, wo der elektrische Widerstand als Funktion der absoluten Temperatur dargestellt ist. Offensichtlich hängen die beiden Knickpunkte bei $\Theta_{1}$ und $\Theta_{2}$ mit CurIE-Punkten zusammen. $\Theta_{2}$ kennen wir schon sehr genau von den Messungen der Anfangssuszeptibilität $\left(\Theta_{2}=697^{\circ} \mathrm{K}\right)$. Nun läßt sich ein zweiter Curie-Punkt $\Theta_{1}$ bei ca. $485^{\circ} \mathrm{K}$ feststellen.

Da der letzte Meßpunkt in Abb. 2 bei $513^{\circ} \mathrm{K}$ sicher oberhalb von $\Theta_{1}$ liegt, ist anzunehmen, daß die entsprechende Sättigungsmagnetisierung zu derjenigen magnetischen Phase gehört, die erst bei $\Theta_{2}=697^{\circ} \mathrm{K}$ ihren Curie-Punkt hat. Es erscheint
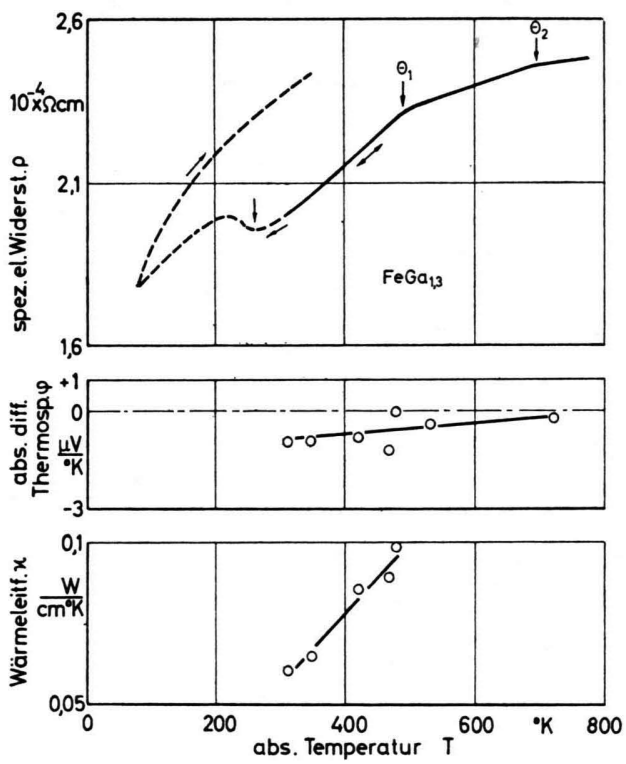

Abb. 3. Elektrischer Widerstand $\varrho$, absolute differentielle Thermospannung $\varphi$ und Wärmeleitfähigkeit $\varkappa$ von $\mathrm{FeGa}_{1,3}$ als Funktion der absoluten Temperatur $T$. 
somit berechtigt, durch diese beiden Punkte die theoretische Idealkurve $M_{\mathrm{s} 2}$ für Spin 1/2

$$
M_{\mathrm{s} 2}=M_{02} \cdot \operatorname{tgh} \frac{M_{\mathrm{s} 2} / M_{02}}{T / \Theta_{2}}
$$

zu legen. Werden diese Werte von der experimentellen Kurve subtrahiert, so erhält man eine zweite gestrichelte Kurve $M_{\mathrm{s} 1}$. Diese gehorcht dem Gesetz

$$
M_{\mathrm{s} 1}=M_{01} \cdot \operatorname{tgh} \frac{M_{\mathrm{s} 1} / M_{01}}{T / \Theta_{1}}
$$

mit $\Theta_{1}=483^{\circ} \mathrm{K}$. Die gemessenen Werte der Sättigungsmagnetisierung unterhalb $\Theta_{1}=483^{\circ} \mathrm{K}$ sind somit im Rahmen der Meßgenauigkeit durch zwei gleichzeitig vorhandene ferromagnetische Phasen 1 und 2 zu erklären. Eine Deutung der experimentellen Ergebnisse in der Weise, daß immer nur eine einzige ferromagnetische Phase vorliegt und bei $\Theta_{1}$ eine Phasenumwandlung stattfindet (analog MnAs), scheint wegen der Kurvenform in Abb. 2 und des Widerstandsverlaufes in Abb. 3 oben ausgeschlossen. Es ist also noch eine Erklärung für das Nebeneinander der beiden ferromagnetischen Strukturen zu finden, obwohl im metallurgischen Sinn keine Mehrphasigkeit vorliegt. Dazu kann man sich vorstellen, daß auf Grund der niedrigen Kristallsymmetrie von $\mathrm{FeGa}_{1,3}$ und der vielen Atome in der Elementarzelle zwei verschiedene ferromagnetische Untergitter existieren, die jedoch nicht wie in Ferrimagneten antiferromagnetisch gekoppelt sind.

5 H. W AGINI, Z. Naturforschg. 19 a, 1541 [1964].
Abb. 3 zeigt den elektrischen Widerstand $\varrho$, die absolute differentielle Thermospannung $\varphi$ und die Wärmeleitfähigkeit $\varkappa$ als Funktion der absoluten Temperatur, gemessen an einer Probe von 2,7 mm $\times 5 \mathrm{~mm} \times 30 \mathrm{~mm}$. Die Messung des elektrischen Widerstandes erfolgte nichtstationär bei einer Temperaturänderung von ca. $5{ }^{\circ} \mathrm{K} / \mathrm{min}$. Die Knick. punkte bei $\Theta_{1}$ und $\Theta_{2}$ wurden schon als CurIEPunkte erkannt. Zu tiefen Temperaturen $(T<260$ ${ }^{\circ} \mathrm{K}$ ) hin fand man einen verschmierten Sprung, wie er bei Gitterumwandlungen zu beobachten ist. Außerdem zeigte sich eine Temperaturhysterese. Der ursprüngliche Zimmertemperaturwert stellte sich nach kurzzeitigem ( 5 min) Glühen bei $800{ }^{\circ} \mathrm{C}$ im Vakuum wieder ein. Dieses Verhalten ist noch durch röntgenographische Analysen zu klären. Magnetische Messungen unterhalb Zimmertemperatur wurden nicht durchgeführt.

Die absolute differentielle Thermospannung $\varphi$ ergab sehr kleine negative Werte.

Wegen des großen elektrischen Widerstandes lag die Wärmeleitfähigkeit auch entsprechend tief. Gemessen wurde nach der stationären Absolutmethode (vgl. ${ }^{5}$ ). Der starke Anstieg mit der Temperatur ist außergewöhnlich.

Herrn Dr. M. Wilhelm danke ich für die Herstellung des Materials und Herrn Dr. H. KandLer für die röntgenographische Untersuchung. Die Kondensatorbatterie wurde freundlicherweise von Herrn H. HieroNymus zur Verfügung gestellt. 